\title{
Effect of sphingosine-1-phosphate and myoblast transplantation on rat acute myocardial infarction
}

\author{
H. Yu' ${ }^{1}$, P.L. Chen ${ }^{2}$, Y. Zhao' ${ }^{1}$ X. Gu ${ }^{3}$, W. He ${ }^{1}$ and Z. Gong ${ }^{2}$ \\ ${ }^{1}$ Key Laboratory of System Biomedicine of Jiujiang University, Jiujiang, \\ Jiangxi Province, China \\ ${ }^{2}$ Department of Student Center, Affiliated Hospital of Jiujiang University, Jiujiang, \\ Jiangxi Province, China \\ ${ }^{3}$ Department of Vasculocardiology, Affiliated Hospital of Jiujiang University, Jiujiang, \\ Jiangxi Province, China \\ Corresponding author: H. Yu \\ E-mail: yuhuan_yh@163.com \\ Genet. Mol. Res. 14 (4): 13843-13851 (2015) \\ Received May 24, 2015 \\ Accepted September 16, 2015 \\ Published October 29, 2015 \\ DOI http://dx.doi.org/10.4238/2015.October.29.4
}

ABSTRACT. In this study, we investigated the effects of sphingosine1-phosphate (S1P) combined with myoblast transplantation on the treatment of acute myocardial infarction and provided a foundation for its clinical application. A rat model of acute myocardial infarction was established by ligating the anterior descending branch of the coronary artery. Serum-free media, myoblasts, myoblasts with S1P liposomes, or myoblasts with liposomes were then injected into the infarcted area. Apoptosis of the transplanted cells was assessed after 24 and $48 \mathrm{~h}$, and changes in heart function and myocardial infarction area were assessed after 4 weeks. After transplantation of S1P into myoblasts, myocardial function was improved compared to that in the other groups. Specifically, the apoptosis of transplanted cells and the area of myocardial infarction decreased significantly $(P<0.01)$, while cardiac function significantly 
improved $(P<0.01)$. The efficacy of S1P and myoblast transplantation on acute myocardial infarction was significantly better than that in the control group (i.e., injection of myoblasts and liposomes) and the serumfree medium group, demonstrating the feasibility of joint S1P and myoblast transplantation for treating myocardial infarction.

Key words: Acute myocardial infarction; Apoptosis; Cell transplantation; Myoblast; Sphingosine-1-phosphate

\section{INTRODUCTION}

Myocardial infarction causes heart failure and death in coronary heart disease patients by playing a key role in muscle cell death (Nadal-Ginard et al., 2003). Cell transplantation therapy can replace necrotized myocardial cells and increase cell number and contraction function to improve heart function; thus, it is a means for gradual treatment of heart disease (Barnett and van den Hoff, 2011). Treatment of myocardial infarction through autologous myoblast transplantation can result in the development of myoblasts into the myocardium in the myocardial infarcted microenvironment. Inclusion body formation is impaired by myocardial function, which is expected to improve heart function, quality of life, and the survival rate of patients (Hagège et al., 2003).

Compared to the use of traditional methods, the use of skeletal muscle myoblasts has been shown to be superior in the treatment of myocardial infarction. However, the use of myoblast transplantation is limited by the death of transplanted cells. Shortly after transplantation in an animal model of low-temperature freezing injury, 50\% of the transplanted cells die (Zhang et al., 2001), thereby reducing the positive effects on cardiac function.

Sphingosine-1-phosphate $(\mathrm{S} 1 \mathrm{P})$ is a bioactive lipid metabolite secreted by a number of blood cells (platelets). This molecule regulates cell proliferation, regeneration, and migration, as well as the movement of intracellular calcium ions, the expression of adhesion molecules, monocyte activation, endothelial cell adhesion, and other biological effects. Recent studies have shown that S1P can significantly reduce the necrosis area caused by ischemia reperfusion injury in myocardial tissue (Egom et al., 2011). In myocardial infarction, aggregation and release of platelets can result in the generation of large amounts of S1P (Sano et al., 2002). S1P can reduce ischemiareperfusion injury and inhibit myocardial cell apoptosis, and has a myocardial protective effect by activating the phosphatidylinositol 3 kinase-Akt signaling pathway (Del Re et al., 2008).

In this study, we combined skeletal muscle myoblast transplantation with S1P injection after establishing a model for acute myocardial infarction in rats. Serum-free media, myoblasts, myoblasts with S1P, and myoblasts with liposomes were injected into the infarcted area, and the mortality of transplanted cells, heart function, myocardial infarction area, and apoptosis of transplanted cells were assessed after 4 weeks. We determined the feasibility of combined S1P and skeletal muscle myoblast treatment of myocardial infarction.

\section{MATERIAL AND METHODS}

\section{Materials and reagents}

Healthy Sprague-Dawley rats weighing $250 \pm 50 \mathrm{~g}$ were obtained from the Experimental 
Animal Center of Jiujiang University. Several key reagents and their providers include the following: S1P (Enzo Biochem, Inc. Plymouth Meeting, New York, USA), phosphatidylcholine (Sigma, St. Louis, MO, USA), and in situ apoptosis detection kit (Promega, Madison, WI, USA).

The instruments and equipment used in this study included a confocal fluorescence microscope (TCS-SP, Leica, Wetzlar, Germany), an electric thermostatic drying oven (DHG-9203A, Shanghai Heng Technology Co. Ltd., Shanghai, China), an automatic high-pressure sterilizing pot (HVE-50, Hirayama Manufacturing Corp., Tokyo, Japan), a $\mathrm{CO}_{2}$ constant temperature cell culture box (type SHELL/JB, Thermo Fisher Scientific Inc., Shanghai, China), an inverted phase contrast microscope (Olympus, Tokyo, Japan), a visible spectrophotometer (type 721, type 723, Shanghai Precision Instrument Co., Ltd., Shanghai, China), a desktop high-speed centrifuge (TGL-16G, Union Instruments, Jintan City, Jiangsu Province, China), a small animal ventilator (HX-100E, Chengdu Technology \& Marker Co., Ltd., Chengdu City, Sichuan Province, China), a BL-420S physiological recorder (Chengdu Technology \& Marker Co. Ltd., and a small animal operation instrument set.

\section{Isolation and culture of skeletal muscle cells}

The isolation and culture of skeletal muscle cells were performed as described by BlancoBose et al. (2001). Briefly, skeletal muscle samples were removed from rat thigh muscle, cut into 1-2-mm fragments, mixed with an enzyme digestion solution $(2.4 \mathrm{U} / \mathrm{mL}$ dispase, $1 \%$ collagenase II, and $2.5 \mathrm{mM} \mathrm{CaCl}$ ) and incubated at $37^{\circ} \mathrm{C}$ for $45 \mathrm{~min}$. The growth media contained Dulbecco's modified Eagle's medium, 50\% Ham F-10, 20\% fetal bovine serum, $2.5 \mathrm{ng} / \mathrm{mL}$ basic fibroblast growth factor, $20 \mathrm{mM} \mathrm{L-glutamine,} \mathrm{and} 1 \%$ penicillin and streptomycin. Cells were inoculated at $5 \mathrm{x}$ $10^{5} \mathrm{cells} / \mathrm{mL}$ on $6-\mathrm{cm}$ Petri dishes coated with $0.1 \%$ poly L-lysine and cultured at $37^{\circ} \mathrm{C}$ in a $5 \% \mathrm{CO}_{2}$ incubator. Using the limited dilution and immunocytochemistry method, the staining-positive and single-cell clones were amplified and cultured.

\section{Construction of the S1P liposome}

The S1P liposome was constructed as described previously by Brailoiu et al. (2002). Briefly, $73 \mu \mathrm{L} 1 \mathrm{mM} \mathrm{S1P}$ was dissolved in $140 \mathrm{mM} \mathrm{KCl}, \mathrm{pH} 6.9$, to prepare a solution of $8.7 \times 10^{-5}$ M $0.833 \mathrm{~mL}$ S1P. Next, $50 \mathrm{mg}$ phosphatidylcholine was dissolved in $2.5 \mathrm{~mL}$ solution containing two ethers. The S1P/KCl solution and the phosphatidylcholine/two ether solution were mixed, and the resulting emulsion was vortexed for $5 \mathrm{~min}$. This emulsion was then placed in a $20^{\circ} \mathrm{C}$ rotary evaporator to evaporate the organic solvent. The resulting solution was dialyzed against a Ringer's solution (110 mM NaCl, $2.5 \mathrm{mM} \mathrm{KCl}, 1.8 \mathrm{mM} \mathrm{CaCl}_{2}, 2 \mathrm{mM}$ Tris- $\mathrm{HCl}, \mathrm{pH}$ 7.2, and $5.6 \mathrm{mM}$ glucose) to remove unbound S1P. Control liposomes that did not contain S1P were prepared in the same manner as was used for the S1P liposomes. The concentration of S1P in the S1P liposome was estimated by using a thin-layer chromatography plate of indene three-ketone coupling reactions.

\section{Preparation of rat model for acute myocardial infarction}

Rats were anesthetized by intraperitoneal injection of $10 \%$ chloral hydrate $(0.4 \mathrm{~mL} / 100$ g) and fixed in the supine position. An electrode was then inserted into the subcutaneous tissue of the extremities, and their electrocardiogram was recorded. In addition, breathing was assisted 
with the aid of a gavage needle, orotracheal intubation, and a small animal ventilator (expiration/ inspiration $=1: 2$, respiratory frequency was 70 breaths $/ \mathrm{min}$, and tidal volume of $15 \mathrm{~mL}$ ). The skin on the left chest was disinfected with povidone iodine, and a horizontal skin incision was made on the left border of sternum (3-4 intercostal). A blunt separation of the muscular layer was made to gain access into the chest from the 3-4 intercostal space, and the pericardium was opened to fully expose the heart. The anterior descending branch of the coronary artery between the left atrial and pulmonary arterial cone was ligated using a 6-0 suture. The electrocardiogram displayed STsegment elevation, indicating that the operation was successful.

\section{Determination of left ventricular function}

A physiological recorder was used to record the left ventricular systolic pressure (LVSP) and end-diastolic pressure (LVEDP). The pressure in the left ventricle was determined as the difference between the 2 measurements mentioned above [i.e., left ventricular developed pressure $($ LVDP $)=$ LVSP - LVEDP]. LVSP and LVDP reflect the left ventricular systolic function, while LVEDP reflects left ventricular diastolic function. The function of the left ventricular cells was assessed before cell transplantation and 4 weeks after transplantation.

\section{Myocardial infarction area}

Ten rats were randomly selected from each group, and the myocardial infarction area was assessed 4 weeks after transplantation. Specifically, the animals were anesthetized as described above, and their hearts were excised. The left and right atria, as well as the right ventricles, were removed and the remaining left ventricle was flushed with phosphate-buffered saline. The left ventricle was divided into 5 parts parallel to the left ventricle long axis, fixed in $4 \%$ formalin, and then embedded with paraffin. Each section was sliced at a thickness of $10 \mu \mathrm{m}$ and stained with 2,3,5-triphenyltetrazolium chloride. The percentage of myocardial infarct area to the total weight of left ventricular was then determined.

\section{Detection of apoptotic cells by in situ end-labeling (terminal deoxynucleotidyl transferase-mediated dUTP-biotin nick end-labeling (TUNEL)}

Apoptotic cells were labeled using the TUNEL method. A confocal fluorescence microscope was used to detect green fluorescent protein-transfected myoblasts with an excitation peak centered at approximately $488 \mathrm{~nm}$. A red fluorescent signal was detected at $>620 \mathrm{~nm}$ for apoptotic nuclei, while a fluorescent signal for DAPI shifted its peak emission wavelength from 475 to 525 $\mathrm{nm}$, with excitation at $360 \mathrm{~nm}$, and showed normal staining of the nuclei. These signals were observed and counted using the single-blind method. At least 200 nuclei (10 random visual fields) were counted for each section (6 cards for each group) using a $40 \mathrm{X}$ objective lens. The apoptosis index was calculated as follows:

$$
\text { Apoptosis index }=\text { number of apoptotic cells } / \text { total cell count } \times 100 \% \quad \text { (Equation 1) }
$$

\section{Experimental groups}

Rats were randomly divided into 4 groups: 1) control group, injected with cell culture fluid 
into the infarct zone ( $N=20), 2)$ myoblast transplantation group, $(\mathrm{N}=20), 3)$ S1P liposomes (1 M) and myoblast transplantation group $(\mathrm{N}=30)$, and 4$)$ muscle cells transplanted into liposomes ( $N$ $=30$ ).

\section{Statistical analysis}

All data were analyzed using the SPSS 11.5 software (SPSS, Inc., Chicago, IL, USA), and the statistical data from each group are reported as means \pm standard deviation. Comparisons between groups were made by single-factor contrast analysis. $P<0.05$ indicates that the difference was statistically significant.

\section{RESULTS}

\section{Identification of myoblast cells by immunocytochemistry in rats}

Myoblast cells positive for desmin immunocytochemical staining are shown in Figure 1.
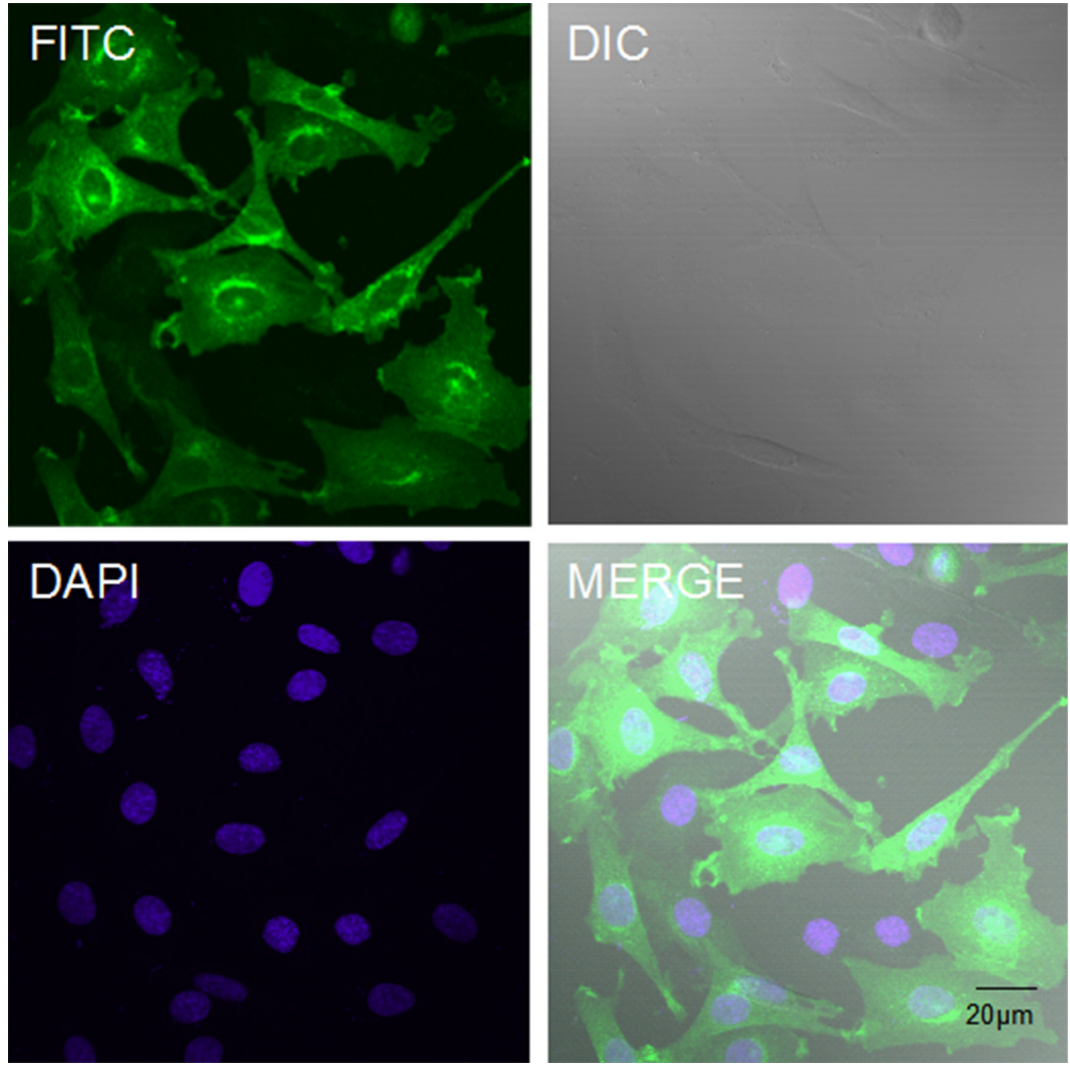

Figure 1. Immunocytochemical staining of myoblast cells. Visualization of desmin in the cytoplasm and nucleus with FITC staining; DAPI staining of the nucleus. DIC shows differential interference contrast imaging; MERGE shows overlap imaging. 


\section{Left ventricular function before and after transplantation}

The function of the left ventricle in the group that received only culture fluid (group 1) showed no difference in LVSP, LVDP, and LVEDP ( $>0.05$; Table 1). The mean values of LVDP and coronary perfusion flow (CPF) in the group that received myoblast injection (group 2) were significantly higher than those of control group (i.e., group that received culture media; $P<0.01$ ). Moreover, in the group that received both myoblasts and S1P liposomes (group 3), the mean values of LVDP and CPF were significantly higher than those in the group that received only myoblasts (group 2; $P<0.01$ ). The mean values of LVDP and CPF showed no significant difference between the group that received myoblasts and liposomes (group 4) and the group that received only myoblasts (group 2; P > 0.05).

\begin{tabular}{|c|c|c|c|c|}
\hline Group & Heart rate (bpm) & LVDP (mmHg) & $\mathrm{CPF}(\mathrm{mL} / \mathrm{min})$ & Infarct size $(\%)$ \\
\hline 1 & $213.01 \pm 22.66$ & $112.44 \pm 4.37$ & $10.35 \pm 0.25$ & $45.72 \pm 1.20$ \\
\hline 2 & $225.86 \pm 26.77$ & $132.01 \pm 12.01^{*}$ & $11.69 \pm 0.14^{*}$ & $38.80 \pm 1.42^{*}$ \\
\hline 3 & $212.12 \pm 28.13$ & $148.69 \pm 3.18^{\#}$ & $12.91 \pm 0.26^{\#}$ & $30.12 \pm 1.91^{\#}$ \\
\hline 4 & $222.33 \pm 29.25$ & $130.01 \pm 10.01^{\$}$ & $11.47 \pm 0.33^{s}$ & $37.60 \pm 1.23^{s}$ \\
\hline
\end{tabular}

\section{Comparison of infarction area in each group before and after transplantation}

Before transplantation, there was no significant difference between groups with respect to the infarction area $(P>0.05)$ (results not shown). Following transplantation, the mean infarction area decreased significantly when the rats received myoblasts (group 2), compared to that observed in the control group (group 1) and the pre-transplantation values $(P<0.01$; Table 1$)$. The mean LVDP and CPF in group 3 were clearly lower than those in group $2(P<0.01)$, while the mean LVDP, CPF, and other indicators showed no significant difference between group 4 and group $2(P>0.05)$.

\section{Apoptosis of myoblasts after transplantation}

The myoblasts were transfected with a green fluorescent protein-containing plasmid before transplantation. After $24 \mathrm{~h}$, these myoblasts were transplanted into the rats with myocardial infarction. The apoptosis of transplanted cells in the group that received S1P liposomes decreased significantly at 24 and $48 \mathrm{~h}$ after transplantation compared to that observed in rats that received only liposomes $(P<0.01$; Figure $2 A, B$ and Figure 3$)$.

\section{DISCUSSION}

With the development of in vitro cell culture, scientists have been attempting to utilize cultured cells to repair damaged myocardium, which may improve heart function. Current research has focused on the use of autologous cell transplantation, which avoids the issue of immune rejection as well as any ethical debates. In particular, autologous skeletal muscle 
A

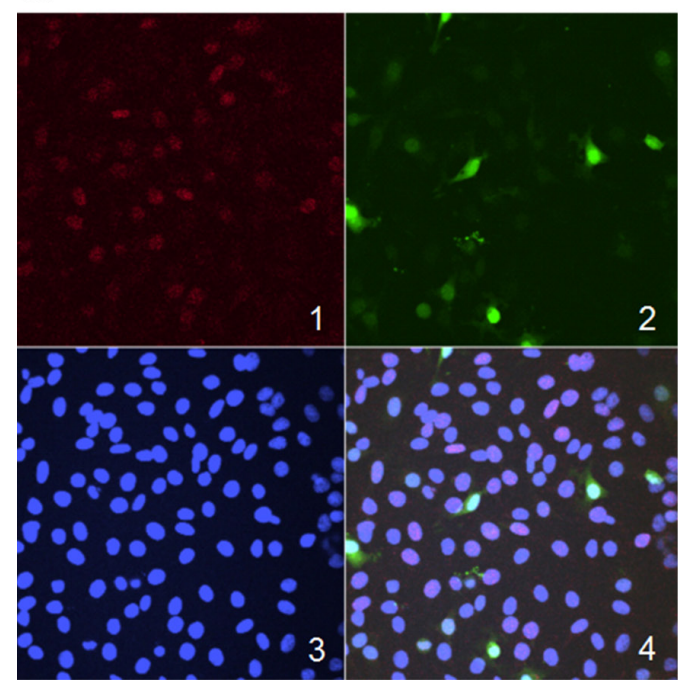

B

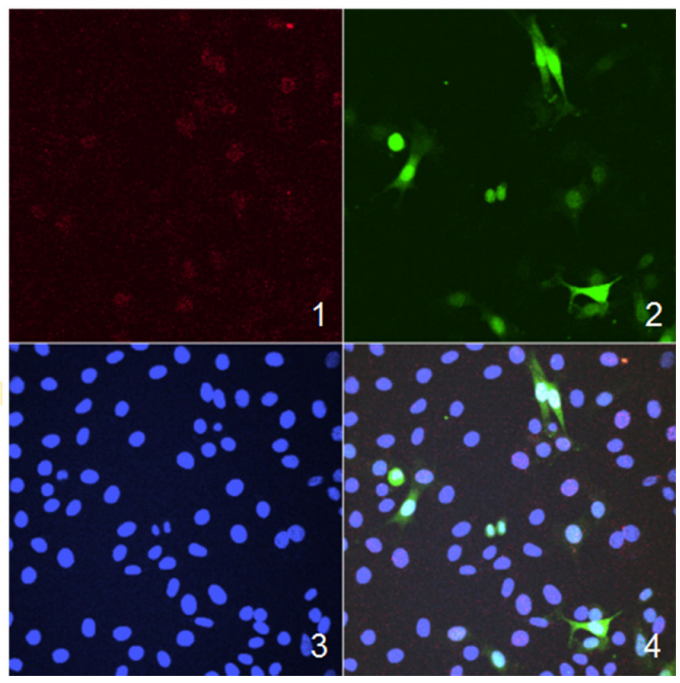

Figure 2. TUNEL-positive cardiomyocytes of all groups under a confocal fluorescence microscope. A. Graft cells are skeletal muscle myoblasts with liposomes and B. graft cells are skeletal muscle myoblasts with liposomal-S1P. (1) TUNEL-positive nuclei, (2) GFP-transfected myoblasts, (3) DAPI-stained nuclei, and (4) merged image of 1-3.

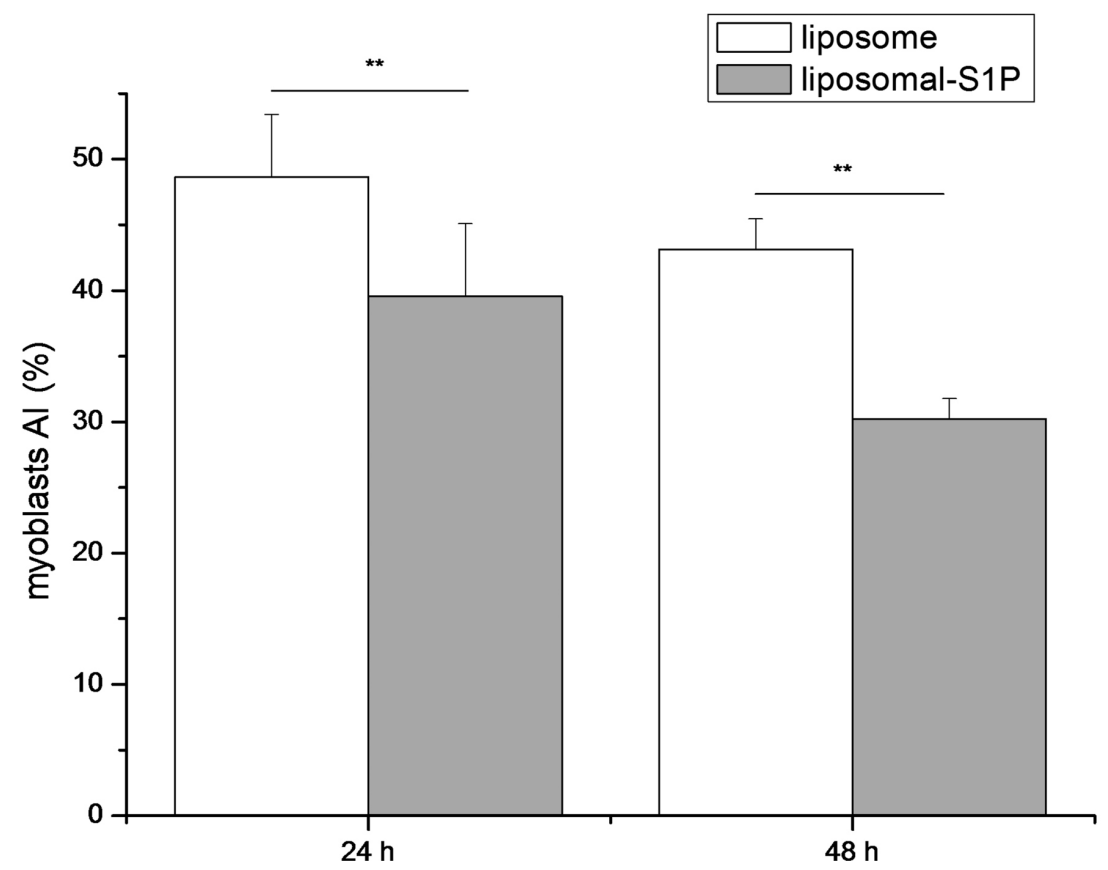

Figure 3. Myoblast $\mathrm{Al}$ at 24 and $48 \mathrm{~h}$ after transplantation with liposomes alone or S1P liposomes. TUNEL-positive myoblasts/total myoblasts $\times 100 \%(\mathrm{Al})$ at 24 and $48 \mathrm{~h}$ after transplantation. Comparisons between the groups were made with single-factor contrast analysis $\left({ }^{*} \mathrm{P}<0.01\right)$. 
cell transplantation has progressed rapidly, and has even been examined in some clinical trials (Menasche et al., 2001; Povsic et al., 2011). However, there are several challenges to autologous skeletal muscle cell transplantation, including the limited supply of these muscle cells and difficulties in their ability to proliferate, differentiate, and form muscle tissue. Therefore, determining how to effectively inhibit apoptosis after transplantation may be essential for improving the efficacy of this treatment.

In this study, we established a model of acute myocardial infarction in rats. The infarcted area of these rats was injected with serum-free media, myoblasts, myoblasts with S1P liposomes, or myoblasts with liposomes. Apoptosis of the transplanted cells was then monitored 24 and 48 $\mathrm{h}$ after transplantation. The results showed that at both time points, the apoptosis of transplanted cells decreased when rats were injected with S1P liposomes compared with that observed in rats that received liposomes (Figure 2 and Figure 3). This suggests that S1P effectively inhibited myoblast apoptosis following transplantation. Test results after 4 weeks showed that cardiac function, myocardial infarction area, and other indicators of rats transplanted with myoblasts and S1P liposomes were significantly improved than those of the group that received myoblasts and liposomes (Table 1). Thus, myoblast transplantation with S1P liposomes effectively improved cardiac function and decreased apoptosis of transplanted cells.

Early reperfusion of myocardial tissue can cause apoptosis and even necrosis. Several animal experiments have confirmed that myocardial cell apoptosis plays an important role in lethal myocardial reperfusion injury (Zhao et al., 2000; Eefting et al., 2004). Therefore, mitigating apoptosis during early reperfusion may be an important factor in preventing significant myocardial reperfusion injury. S1P is mainly catalyzed by sphingosine kinase 1 , which plays an important role in the process of ischemic preadaptation. The inhibition of sphingosine kinase 1 activity weakens its protective effect on the heart (Kennedy et al., 2009). S1P is mediated by a subfamily of $G$ protein-coupled receptors encoded by endothelial differentiation genes (Edgs). In the myocardium, Edg-3 and Edg-5 receptors are expressed at high levels (Landeen et al., 2008). S1P can protect myocardial cells by binding to Edg-3 and Edg-5 receptors, which activates Akt and reduces ischemia/reperfusion injury (Means et al., 2007).

The results showed that S1P inhibited the apoptosis of transplanted cells. These results agree with those of a large number of studies demonstrating that S1P reduced ischemia/reperfusion injury and protected myocardial cells. This study provides a theoretical and experimental basis for the subsequent transplantation of skeletal myoblasts combined with exogenous S1P for treating ischemic diseases.

\section{Conflicts of interest}

The authors declare no conflict of interest.

\section{ACKNOWLEDGMENTS}

Research supported by the National Youth Science Fund Project (\#81000075), the National Natural Science Fund Project (\#81460048), the Jiangxi Province Natural Science Fund Project (\#20142BAB215031), the Key Scientific Research Project of Education Department of Jiangxi Province (\#GJJ11694), the Research Project of Education Department of Jiangxi Province (\#GJJ14720), and the China Scholarship Council (CSC). 


\section{REFERENCES}

Barnett P and van den Hoff MJ (2011). Cardiac regeneration: different cells same goal. Med. Biol. Eng. Comput. 49: 723-732.

Blanco-Bose WE, Yao CC, Kramer RH and Blau HM (2001). Purification of mouse primary myoblasts based on alpha 7 integrin expression. Exp. Cell Res. 265: 212-220.

Brailoiu E, Cooper RL and Dun NJ (2002). Sphingosine 1-phosphate enhances spontaneous transmitter release at the frog neuromuscular junction. Br. J. Pharmacol. 136: 1093-1097.

Del Re DP, Miyamoto S and Brown JH (2008). Focal adhesion kinase as a RhoA-activable signaling scaffold mediating Akt activation and cardiomyocyte protection. J. Biol. Chem. 283: 35622-35629.

Eefting F, Rensing B, Wigman J, Pannekoek WJ, et al. (2004). Role of apoptosis in reperfusion injury. Cardiovasc. Res. 61: 414-426.

Egom EE, Mohamed TM, Mamas MA, Shi Y, et al. (2011). Activation of Pak1/Akt/eNOS signaling following sphingosine-1phosphate release as part of a mechanism protecting cardiomyocytes against ischemic cell injury. Am. J. Physiol. Heart Circ. Physiol. 301: H1487-H1495.

Hagège AA, Carrion C, Menasché P, Vilquin JT, et al. (2003). Viability and differentiation of autologous skeletal myoblast grafts in ischemic cardiomyopathy. Lancet 361: 491-492.

Kennedy S, Kane KA, Pyne NJ and Pyne S (2009). Targeting sphingosine-1-phosphate signaling for cardioprotection. Curr. Opin. Pharmacol. 9: 194-201.

Landeen LK, Dederko DA, Kondo CS, Hu BS, et al. (2008). Mechanisms of the negative inotropic effects of sphingosine-1phosphate on adult mouse ventricular myocytes. Am. J. Physiol. Heart Circ. Physiol. 294: H736-H749.

Means CK, Xiao CY, Li Z, Zhang T, et al. (2007). Sphingosine-1-phosphate S1P2 and S1P3 receptor-mediated Akt activation protects against in vivo myocardial ischemia-reperfusion injury. Am. J. Physiol. Heart Circ. Physiol. 292: H2944-H2951.

Menasche P, Hagège AA, Scorsin M, Pouzet B, et al. (2001). Myoblast transplantation for heart failure. Lancet 357: 279-280.

Nadal-Ginard B, Kajstura J, Leri A and Anversa P (2003). Myocyte death, growth, and regeneration in cardiac hypertrophy and failure. Circ. Res. 92: 139-150.

Povsic TJ, O'Connor CM, Henry T, Taussig A, et al. (2011). A double-blind, randomized, controlled, multicenter study to assess the safety and cardiovascular effects of skeletal myoblast implantation by catheter delivery in patients with chronic heart failure after myocardial infarction. Am. Heart J. 162: 654-662 e651.

Sano T, Baker D, Virag T, Wada A, et al. (2002). Multiple mechanisms linked to platelet activation result in lysophosphatidic acid and sphingosine-1-phosphate generation in blood. J. Biol. Chem. 277: 21197-21206.

Zhang M, Methot D, Poppa V, Fujio Y, et al. (2001). Cardiomyocyte grafting for cardiac repair: graft cell death and anti-death strategies. J. Mol. Cell Cardiol. 33: 907-921.

Zhao ZQ, Nakamura M, Wang NP, Wilcox JN, et al. (2000). Reperfusion induces myocardial apoptotic cell death. Cardiovasc. Res. 45: 651-660. 